\title{
ANALYTIC ISOMORPHISMS OF COMPRESSED LOCAL ALGEBRAS
}

\author{
J. ELIAS AND M. E. ROSSI \\ (Communicated by Irena Peeva)
}

\begin{abstract}
In this paper we consider Artin compressed local algebras, that is, local algebras with maximal length in the class of those with given embedding dimension and socle type. They have been widely studied by several authors, including Boij, Iarrobino, Fröberg and Laksov. In this class the Gorenstein algebras play an important role. The authors proved that a compressed Gorenstein $K$-algebra of socle degree 3 is canonically graded, i.e. analytically isomorphic to its associated graded ring. This unexpected result has been extended to compressed level $K$-algebras of socle degree 3 in a paper by De Stefani. This paper somehow concludes the investigation proving that Artin compressed Gorenstein $K$-algebras of socle degree $s \leq 4$ are always canonically graded, but explicit examples prove that the result does not extend to socle degree 5 or to compressed level $K$-algebras of socle degree 4 and type $>1$. As a consequence of this approach we present classes of Artin compressed $K$-algebras which are canonically graded.
\end{abstract}

\section{Compressed Algebras AND INVERse System}

Let $A=R / I$ be an Artin local ring where $R=K\left[\left[x_{1}, \ldots, x_{n}\right]\right]$ is the formal power series ring and $K$ is an algebraically closed field of characteristic zero. We denote by $\mathfrak{m}=\mathcal{M} / I$ the maximal ideal of $A$ where $\mathcal{M}=\left(x_{1}, \ldots, x_{n}\right)$ and let $\operatorname{Soc}(A)=0: \mathfrak{m}$ be the socle of $A$. Throughout this paper we denote by $s$ the socle degree of $A$, that is, the maximum integer $j$ such that $\mathfrak{m}^{j} \neq 0$. The type of $A$ is $t:=\operatorname{dim}_{K} \operatorname{Soc}(A)$.

Let $A$ be of initial degree $v$, that is, $I \subseteq \mathcal{M}^{v} \backslash \mathcal{M}^{v+1}$ and socle degree $s$. The socle type $E=E(A)=\left(0, \ldots, e_{v-1}, e_{v}, \ldots, e_{s}, 0,0, \ldots\right)$ of $A$ is the sequence $E$ of natural numbers where

$$
e_{i}:=\operatorname{dim}_{K}\left((0: \mathfrak{m}) \cap \mathfrak{m}^{i} /(0: \mathfrak{m}) \cap \mathfrak{m}^{i+1}\right) .
$$

Clearly $e_{s}>0$ and $e_{j}=0$ for $j>s$, but other conditions are necessary on $E$ for being permissible for an Artin $K$-algebra of given initial degree $v$ and socle degree $s$ (see [10]). Essentially the conditions require room for generators of $I$ of valuation $v$ and assure that there are not generators of valuation $v-1$.

A level algebra $A$ of socle degree $s$ (say also $s$-level), type $t$ and embedding dimension $n$ is an Artin quotient of $R$ whose socle is concentrated in a single degree

Received by the editors August 6, 2012 and, in revised form, July 2, 2013.

2010 Mathematics Subject Classification. Primary 13H10; Secondary 13H15, 14C05.

The first author was partially supported by MTM2013-40775-P.

The second author was partially supported by PRIN 2010-11 "Geometria delle varieta' algebriche". 
(i.e. $\operatorname{Soc}(A)=\mathfrak{m}^{s}$ ) and satisfying $\operatorname{dim}_{K} \operatorname{Soc}(A)=t$. Hence $e_{j}=0$ for $j \neq s$ and $e_{s}=t$. The Artin algebra is Gorenstein if $t=1$.

It makes sense to consider maximum length Artin algebras of given socle type $E$ and they can be characterized in terms of their Hilbert function. The Hilbert vector of $A$ is denoted by $\operatorname{HF}(A)=\left\{h_{0}, h_{1}, \ldots, h_{s}\right\}$ where $h_{i}=h_{i}(A)=\operatorname{dim}_{K} \mathfrak{m}^{i} / \mathfrak{m}^{i+1}$ is the Hilbert function of $A$. By its definition, the Hilbert function of $A$ coincides with the Hilbert function of the corresponding associated graded ring $g r_{\mathfrak{m}}(A)=$ $\bigoplus_{i>0} \mathfrak{m}^{i} / \mathfrak{m}^{i+1}$. We say that the Hilbert function $H F(A)$ is maximal in the class of Artin level algebras of given embedding dimension and socle type, if for each integer $i, h_{i}(A) \geq h_{i}\left(A^{\prime}\right)$ for any other Artin algebra $A^{\prime}$ in the same class. The existence of a maximal $H F(A)$ was shown for graded algebras by A. Iarrobino [10. In the general case it was shown by Fröberg and Laksov [9] and by Emsalem [8] and by A. Iarrobino and the first author in [5] in the local case.

Definition 1.1. An Artin algebra $A=R / I$ of socle type $E$ is compressed if and only if it has maximal length $e(A)=\operatorname{dim}_{K} A$ among Artin quotients of $R$ having socle type $E$ and embedding dimension $n$.

The maximality of the Hilbert function characterizes compressed algebras as follows. If $A$ is an Artin algebra (graded or local) of socle type $E$, it is known that for $i \geq 0$,

$$
h_{i}(A) \leq \min \left\{\operatorname{dim}_{K} R_{i}, e_{i} \operatorname{dim}_{K} R_{0}+e_{i+1} \operatorname{dim}_{K} R_{1}+\cdots+e_{s} \operatorname{dim}_{K} R_{s-i}\right\} .
$$

Accordingly with [10, Definition 2.4. B], we can rephrase the previous definition in terms of the Hilbert function.

Definition 1.2. A local (or graded) $K$-algebra $A$ of socle degree $s$, socle type $E$ and initial degree $v$ is compressed if

$$
h_{i}(A)= \begin{cases}\sum_{u=i}^{s} e_{u}\left(\operatorname{dim}_{K} R_{u-i}\right) & \text { if } i \geq v, \\ \operatorname{dim}_{K} R_{i} & \text { otherwise. }\end{cases}
$$

In particular an $s$-level algebra $A$ of type $t$ and embedding dimension $n$ is compressed if

$$
h_{i}(A)=\min \left\{\left(\begin{array}{c}
n+i-1 \\
i
\end{array}\right), t\left(\begin{array}{c}
n+s-i-1 \\
s-i
\end{array}\right)\right\} .
$$

If $t=1$ and the above equality holds, then $A$ is called compressed Gorenstein algebra or also extremal Gorenstein algebra.

It is clear that compressed algebras impose several restrictive numerical conditions on the socle sequence $E$ (see [10], Definition 2.2). For instance if $v$ is the initial degree of $A$, then

$$
e_{v-1}=\max \left\{0, \operatorname{dim}_{K} R_{v-1}-\sum_{u \geq v}\left(e_{u} \operatorname{dim}_{K} R_{u-(v-1)}\right)\right\} .
$$

If $s \geq 2(v-1)$, then it is easy to see that $e_{v-1}=0$ because $\operatorname{dim}_{K} R_{s-(v-1)} \geq$ $\operatorname{dim}_{K} R_{v-1}$. This is the case if $A$ is Gorenstein.

Y. H. Cho and I. Iarrobino proved that the family of graded compressed level quotients of $P=K\left[x_{1}, \ldots, x_{n}\right]$ is parameterized by an open dense subset of the Grassmanian of codimension $c$ quotients of $P_{s}$ and has dimension $c\left(\operatorname{dim} P_{s}-c\right)$ (see [3]). Boij studied the minimal free resolutions for compressed graded level 
algebras [1] and Stanley and Hibi have studied connections of these algebras with combinatorics.

Accordingly with Emsalem [8, p. 408] we give the following definition.

Definition 1.3. A local algebra $(A, \mathfrak{m})$ is canonically graded if there exists a $K$ algebra isomorphism between $A$ and its associated graded ring $g r_{\mathfrak{m}}(A)$.

As a consequence, $A$ is canonically graded if and only if $A$ is analytically isomorphic to any standard graded $K$-algebra. Remark that a local $K$-algebra can be considered graded in a non-standard way, but not canonically graded accordingly with the above definition. For instance, consider $A=K\left[\left[t^{2}, t^{3}\right]\right]$; in this case $A$ is not analytically isomorphic to $\operatorname{gr}_{\mathfrak{m}}(A) \cong K[x, y] /\left(y^{2}\right)$ because $A$ is reduced and $g r_{\mathfrak{m}}(A)$ is not reduced, but we remark that $A \cong K[x, y] /\left(y^{2}-x^{3}\right)$ is graded setting $\operatorname{deg}(x)=2$ and $\operatorname{deg}(y)=3$.

Actually it is very rare that a local ring is isomorphic to its associated graded ring. If this is the case, there can be several applications; see for example [2, 6], 7].

If $A$ is an Artin local (or graded) ring, the dual module $V=\operatorname{Hom}(A, K)$ can be identified with a maximal length submodule of the polynomial $\operatorname{ring} P=$ $K\left[y_{1}, \ldots, y_{n}\right]$ which is closed under partial derivation. We recall the main facts and establish notation concerning the inverse system of Macaulay in the study of Artin local rings $(A, \mathfrak{m})$. The reader should refer to [8] and [11 for an extended treatment. It is known that $P$ has an $R$-module structure under the following action:

$$
\begin{aligned}
\circ: \quad R \times P & \longrightarrow P \\
(f, g) & \rightarrow f \circ g=f\left(\partial_{y_{1}}, \ldots, \partial_{y_{n}}\right)(g)
\end{aligned}
$$

where $\partial_{y_{i}}$ denotes the partial derivative with respect to $y_{i}$. If we denote by $x^{\alpha}=$ $x_{1}^{\alpha_{1}} \cdots x_{n}^{\alpha_{n}}$ and $y^{\beta}=y_{1}^{\beta_{1}} \cdots y_{n}^{\beta_{n}}$, then

$$
x^{\alpha} \circ y^{\beta}= \begin{cases}\frac{\beta !}{(\beta-\alpha) !} y^{\beta-\alpha} & \text { if } \beta_{i} \geq \alpha_{i} \text { for } i=1, \cdots, n, \\ 0 & \text { otherwise }\end{cases}
$$

where $\frac{\beta !}{(\beta-\alpha) !}=\prod_{i=1}^{n} \frac{\beta_{i} !}{\left(\beta_{i}-\alpha_{i}\right) !}$. Starting from $\circ$ we consider the exact pairing of $K$-vector spaces:

$$
\begin{aligned}
& \langle,\rangle: R \times P \quad \longrightarrow \quad K \\
& (f, g) \rightarrow(f \circ g)(0) .
\end{aligned}
$$

If $S \subseteq P$, then its annihilator in $R$ is $A n n_{R}(S)=\{f \in R: f \circ g=0$ for all $g \in$ $S$ \}. The $R$-submodule generated by $S$ in $P$ is closed under partial derivation. Then $A n n_{R}(S)$ is an ideal of $R$. Conversely, for any ideal $I \subset R$ we define the following $R$-submodule of $P$ :

$$
I^{\perp}:=\{g \in P \mid f \circ g=0 \quad \forall f \in I\}=\{g \in P \mid\langle f, g\rangle=0 \quad \forall f \in I\} .
$$

Starting from an Artin local algebra $R / I$ with socle degree $s, I^{\perp}$ is generated by polynomials of degree $\leq s$.

If $S$ is generated by a sequence $\underline{G}:=G_{1}, \ldots, G_{t}$ of polynomials of $P$, then we will write $A n n_{R}(\underline{G})$ and

$$
A_{\underline{G}}=R / A n n_{R}(\underline{G})
$$

where $A n n_{R}(\underline{G})=\bigcap_{j=1}^{t} A n n_{R}\left(G_{j}\right)$. If $A_{\underline{G}}$ is compressed, then we may assume that each $A_{G_{j}}$ is an extremal Gorenstein algebra. Notice that even if each $A_{G_{j}}$ is 
canonically graded, then $A_{\underline{G}}$ is not necessarily canonically graded because a uniform analytic isomorphism may not exist (see Example 3.5).

F. S. Macaulay in 12 proved that there exists a one-to-one correspondence between ideals $I \subseteq R$ such that $R / I$ is an Artin local ring and $R$-submodules $M$ of $P$ which are finitely generated; see also Emsalem [8, Section B, Proposition 2] and Iarrobino [11, Lemma 1.2]. In this correspondence, Artin $K$-algebras $A=R / I$ of embedding dimension $n$, socle degree $s$ and type $t$ correspond to $R$-submodules of $P$ generated by $t$ polynomials $G_{1}, \ldots, G_{t}$ of degree $d_{i}$ (depending on the socle type) such that the leading forms $G_{1}\left[d_{1}\right], \ldots, G_{t}\left[d_{t}\right]$ of degree $d_{i}$ (write $G_{i}=G_{i}\left[d_{i}\right]+$ lower terms...) are linearly independent. If $A=A_{\underline{G}}$ where $\underline{G}:=G_{1}, \ldots, G_{t}$ is an $s$-level local algebra of type $t\left(d_{i}=s\right.$ for all $\left.i=1, \ldots, t\right)$, then the associated graded ring $g r_{\mathfrak{m}}(A)$ is not necessarily level and in general $\operatorname{dim}_{K} \operatorname{Soc}\left(\operatorname{gr} r_{\mathfrak{m}}(A)\right) \geq t$. Actually the graded $K$-algebra

$$
Q=P / A n n_{P}(\underline{G}[s])
$$

(where $\underline{G}[s]:=G_{1}[s], \ldots, G_{t}[s]$ ) is a graded $s$-level algebra of type $t$ and it is the unique quotient of $g r_{\mathfrak{m}}(A)$ with socle degree $s$ and type $t$. In particular $g r_{\mathfrak{m}}(A)$ is level if and only if $g r_{\mathfrak{m}}(A) \simeq Q$ (see [4]).

The following result was proved in [10, Proposition 3.7 and Corollary 3.8].

Proposition 1.4. The compressed local algebra $A$ whose dual module is generated by $G_{1}, \ldots, G_{t}$ of degrees $d_{1}, \ldots, d_{t}$ has a compressed associated graded ring $g r_{\mathfrak{m}}(A)$ whose dual module is generated by $G_{1}\left[d_{1}\right], \ldots, G_{t}\left[d_{t}\right]$, the leading forms of $G_{1}, \ldots, G_{t}$. Conversely if $g r_{\mathfrak{m}}(A)$ is compressed, then $A$ is compressed and $E(A)=$ $E\left(g r_{\mathfrak{m}}(A)\right)$.

It is possible to compute the Hilbert function of $A=R / I$ via the inverse system. We define the following $K$-vector space:

$$
\left(I^{\perp}\right)_{i}:=\frac{I^{\perp} \cap P_{\leq i}+P_{<i}}{P_{<i}} .
$$

Then it is known that

$$
h_{i}(R / I)=\operatorname{dim}_{K}\left(I^{\perp}\right)_{i} .
$$

Notice that in the graded setting $A=R / \operatorname{Ann}(\underline{G})$,

$$
h_{i}(R / I)=\operatorname{dim}_{K}\left(I^{\perp}\right)_{i}=\operatorname{dim}_{K}\left\langle\partial_{s-i} \underline{G}\right\rangle
$$

where $\partial_{s-i}$ denotes the partial derivatives of order $s-i$. We will translate the above equality in terms of the rank of suitable matrices associated to $\underline{G}$.

Let $\Omega=\left\{\omega_{i}\right\}$ be the canonical basis of $R / \mathcal{M}^{s+1}$ as a $K$-vector space consisting of the standard monomials $x^{\alpha}$ ordered by the deg-lex order with $x_{1}>\cdots>x_{n}$. Then the dual basis with respect to the action $\circ$ is the basis $\Omega^{*}=\left\{\omega_{i}^{*}\right\}$ of $P_{\leq j}$ where

$$
\left(x^{\alpha}\right)^{*}=\frac{1}{\alpha !} y^{\alpha} .
$$

In fact $\omega_{j} \circ \omega_{i}^{*}=\delta_{i j}$, where $\delta_{i j}=0$ if $i \neq j$ and $\delta_{i i}=1$.

Given a form $G$ of degree $s$ and an integer $q \leq s$, we denote by $\Delta^{q}(G)$ the $\left(\begin{array}{c}n-1+s-q \\ n-1\end{array}\right) \times\left(\begin{array}{c}n-1+q \\ n-1\end{array}\right)$ matrix whose columns are the coordinates of $\partial_{\underline{i}}(G),|\underline{i}|=q$, with respect to $\left(x^{L}\right)^{*}=\frac{1}{L !} y^{L},|L|=s-q(\underline{i}$ and $L$ are $n$-uples of integers $)$. We will denote by $(L, \underline{i})$ the corresponding position in the matrix $\Delta^{q}(G)$. In the following $L+\underline{i}$ denotes the sum in $\mathbb{N}^{n}$. 
Proposition 1.5. Let $G \in P=K\left[y_{1}, \cdots, y_{n}\right]$ be a form of degree s. Then

$$
h_{s-i}\left(A_{G}\right)=\operatorname{rank}\left(\Delta^{i}(G)\right) \leq \min \left\{\left(\begin{array}{c}
n-1+s-i \\
n-1
\end{array}\right),\left(\begin{array}{c}
n-1+i \\
n-1
\end{array}\right)\right\}
$$

for $i=0, \cdots, s$. The equality holds if and only if $A_{G}$ is compressed.

Proof. It is a straight consequence of (3) and (4), and the definition of the matrix $\Delta^{i}(G)$ of size $\left(\begin{array}{c}n-1+i \\ n-1\end{array}\right) \times\left(\begin{array}{c}n-1+s-i \\ n-1\end{array}\right)$.

Proposition 1.6. Let $G=\sum_{|\underline{j}|=s} \beta_{\underline{j}} \frac{1}{j !} y^{\underline{j}}$ be a degree s homogeneous polynomial. Given an integer $q$, the $(L, \underline{i})$-component of $\Delta^{q}(G),|L|=s-q,|\underline{i}|=q$, is

$$
\Delta^{q}(G)_{(L, \underline{i})}=\beta_{L+\underline{i}} .
$$

Proof. Let us consider the derivative

$$
\partial_{\underline{i}}(G)=\sum_{|\underline{j}|=s, \underline{j} \geq \underline{i}} \beta_{\underline{j}} \frac{1}{(\underline{j}-\underline{i}) !} y^{\underline{j}-\underline{i}} .
$$

$|\underline{i}|=q ; \underline{j} \geq \underline{i}$ means that $j_{w} \geq i_{w}$ for $w=1, \cdots, n$. If we write $L=\underline{j}-\underline{i}$, then

$$
\partial_{\underline{i}}(G)=\sum_{|L|=s-q} \beta_{L+\underline{i}} \frac{1}{L !} y^{L} .
$$

From this we get the claim.

It is easy to deduce the following corollary which gives via (5) an alternative proof of the fact that a graded Gorenstein algebra $A_{G}$ has symmetric Hilbert function.

Corollary 1.7. Let $G$ be a form of $P$ of degree s. Given an integer $i \leq s$,

$$
\Delta^{i}(G)={ }^{\tau} \Delta^{s-i}(G)
$$

where $^{\tau}$ denotes the transpose matrix.

With the previous notation, let $A_{\underline{G}}$ be a graded level algebra. We can define for all integers $i \leq s$,

$$
\Delta^{i}(\underline{G})=\left(\begin{array}{c}
\frac{\Delta^{i}\left(G_{1}\right)}{\vdots} \\
\frac{\Delta^{i}\left(G_{t}\right)}{2}
\end{array}\right)
$$

which is a $t\left(\begin{array}{c}n-1+s-i \\ n-1\end{array}\right) \times\left(\begin{array}{c}n-1+i \\ n-1\end{array}\right)$ matrix. We get the following result.

Proposition 1.8. Let $A=A_{\underline{G}}$ be a compressed s-level local algebra of type t. Then for every $i=1, \ldots, s$

$$
h_{i}(A)=\operatorname{rank}\left(\Delta^{i}(\underline{G}[s])\right)=\min \left\{\left(\begin{array}{c}
n-1+i \\
n-1
\end{array}\right), t\left(\begin{array}{c}
n-1+s-i \\
n-1
\end{array}\right)\right\} .
$$

Proof. By Proposition 1.4 we know that $g r_{\mathfrak{m}}(A)$ is level compressed of socle degree $s$ and type $t$. Since $g r_{\mathfrak{m}}(A)$ is level if and only if $g r_{\mathfrak{m}}(A) \simeq Q=P / \operatorname{Ann}(\underline{G}[s])$, the result follows by Proposition 1.5 . 


\section{Automorphisms of Artin local algebras and associated matrices}

Given a $K$-algebra $C$, we will denote by $A u t(C)$ the group of the automorphisms of $C$ as a $K$-algebra and by $A u t_{K}(C)$ as a $K$-vector space. The automorphisms of $R$ as a $K$-algebra are well known. They act as a replacement of $x_{i}$ by $z_{i}$, $i=1, \cdots, n$, such that $\mathcal{M}=\left(x_{1}, \ldots, x_{n}\right)=\left(z_{1}, \ldots, z_{n}\right)$. Actually, since $\mathcal{M}^{s+1} \subseteq I$, we are interested in the automorphisms of $R / \mathcal{M}^{s+1}$ of $K$-algebras induced by the projection $\pi: R \longrightarrow R / \mathcal{M}^{s+1}$. Clearly $\operatorname{Aut}\left(R / \mathcal{M}^{s+1}\right) \subseteq A u t_{K}\left(R / \mathcal{M}^{s+1}\right)$. For all $p \geq 1, I_{p}$ denotes the identity matrix of order $\left(\begin{array}{c}n+p-1 \\ p\end{array}\right)$.

For any $\varphi \in A u t_{K}\left(R / \mathcal{M}^{s+1}\right)$ we may associate a matrix $M(\varphi)$ with respect to the basis $\Omega$ of size $r=\operatorname{dim}_{K}\left(R / \mathcal{M}^{s+1}\right)=\left(\begin{array}{c}n+s \\ s\end{array}\right)$ already defined. Given $I$ and $J$ ideals of $R$ such that $\mathcal{M}^{s+1} \subset I, J$, there exists an isomorphism of $K$-algebras

$$
\varphi: R / I \rightarrow R / J
$$

if and only if $\varphi$ is canonically induced by a $K$-algebra automorphism of $R / \mathcal{M}^{s+1}$ sending $I / \mathcal{M}^{s+1}$ to $J / \mathcal{M}^{s+1}$. In particular $\varphi$ is an isomorphism of $K$-vector spaces. We denote the dual of a $K$-vector space $V$ by $V^{*}$. Dualizing

$$
\varphi^{*}:(R / J)^{*} \rightarrow(R / I)^{*}
$$

is an isomorphism of the $K$-vector subspaces where $(R / I)^{*} \simeq I^{\perp}$ and $(R / J)^{*} \simeq J^{\perp}$ of $P_{\leq s}$ according to the exact pairing (2). Hence ${ }^{t} M(\varphi)$ is the matrix associated to $\varphi^{*}$ with respect to the basis $\Omega^{*}$ of $P_{\leq s}$.

We denote by $\mathcal{R}$ the subgroup of $A u t_{K}\left(P_{\leq s}\right)$ (automorphisms of $P_{\leq s}$ as a $K$ vector space) represented by the matrices ${ }^{\tau} M(\varphi)$ of $G l_{r}(K)$ with $\varphi \in A u t\left(R / \mathcal{M}^{s+1}\right)$.

By Emsalem, 8, Proposition 15], the classification, up to analytic isomorphism, of the Artin local $K$-algebras of multiplicity $d$, socle degree $s$ and embedding dimension $n$ is equivalent to the classification, up to the action of $\mathcal{R}$, of the $K$ vector subspaces of $P_{\leq s}$ of dimension $d$, stable by derivations and containing $P_{\leq 1}=$ $K\left[y_{1}, \ldots, y_{n}\right]_{\leq 1}$.

Our goal is to translate the study of analytic isomorphisms of Artin level $K$ algebras $A=R / I$ of socle $s$ and type $t$ in terms of the corresponding dual polynomials of degree $s$ in $P$ in an effective computational framework.

This section is quite technical and the idea is the generalization of the method used in [6]. The main goal is Corollary 2.3. One could observe that, in view of it, the machinery could be presented assuming $s \leq 4$. Actually the presentation does not improve substantially and one loses the control of the general case that could be useful for further investigations.

Let $\underline{F}=F_{1}, \ldots, F_{t}$, respectively $\underline{G}=G_{1}, \ldots, G_{t}$, be polynomials of degree $s$. Let $\varphi \in \operatorname{Aut}\left(R / \mathcal{M}^{s+1}\right)$. From the previous facts we have

$$
\varphi\left(A_{\underline{F}}\right)=A_{\underline{G}} \quad \text { if and only if }\left(\varphi^{*}\right)^{-1}\left(\langle\underline{F}\rangle_{R}\right)=\langle\underline{G}\rangle_{R} .
$$

If $F_{i}=b_{i 1} \omega_{1}^{*}+\cdots+b_{i r} \omega_{r}^{*} \in P_{\leq s}$, then we will denote the row vector of the coefficients of the polynomial with respect to the basis $\Omega^{*}$ by

$$
\left[F_{i}\right]_{\Omega^{*}}=\left(b_{i 1}, \ldots, b_{i r}\right) .
$$

If there exists $\varphi \in \operatorname{Aut}\left(R / \mathcal{M}^{s+1}\right)$ such that

$$
\left[G_{i}\right]_{\Omega^{*}} M(\varphi)=\left[F_{i}\right]_{\Omega^{*}} \text {, for every } i=1, \ldots, t \text {, then } \varphi\left(A_{\underline{F}}\right)=A_{\underline{G}} .
$$

Let $s$ be a positive integer. The aim of this section is to provide a structure of the matrix $M(\varphi)$ associated to special $K$-algebra isomorphisms $\varphi \in A u t\left(R / \mathcal{M}^{s+1}\right)$. 
Let $\varphi_{s-p}$ be an automorphism of $R / \mathcal{M}^{s+1}$ such that $\varphi_{s-p}=I d$ modulo $\mathfrak{m}^{p+1}$, with $1 \leq p \leq s$, that is,

$$
\varphi_{s-p}\left(x_{j}\right)=x_{j}+\sum_{|\underline{i}|=p+1} a_{\underline{i}}^{j} x^{\underline{i}}+\text { higher terms }
$$

for $j=1, \ldots, n$ and $a_{i}^{j} \in K$ for each $n$-uple $\underline{i}$ such that $|\underline{i}|=p+1$. In the following we will denote $\underline{a}:=\left(a_{\underline{i}}^{1},|\underline{i}|=p+1 ; \cdots ; a_{\underline{i}}^{n},|\underline{i}|=p+1\right) \in K^{n\left(\begin{array}{c}n+p \\ n-1\end{array}\right)}$.

The matrix associated to $\varphi_{s-p}$, say $M\left(\varphi_{s-p}\right)$, is an element of $G l_{r}(K), r=\left(\begin{array}{c}n+s \\ s+1\end{array}\right)$, with respect to the basis $\Omega$ of $R / \mathcal{M}^{s+1}$. We write $M\left(\varphi_{s-p}\right)=\left(B_{i, j}\right)_{0 \leq i, j \leq s}$ where $B_{i, j}$ is an $\left(\begin{array}{c}n+i-1 \\ i\end{array}\right) \times\left(\begin{array}{c}n+j-1 \\ j\end{array}\right)$ matrix of the coefficients of monomials of degree $i$ appearing in $\varphi\left(x^{\underline{j}}\right)$ where $\underline{j}=\left(j_{1}, \ldots, j_{n}\right)$ such that $|\underline{j}|=j$.

It is easy to verify that:

$$
B_{i, j}=\left\{\begin{array}{l}
0, \quad 0 \leq i<j \leq s, \text { or } j=1, i=1, \cdots, s, \\
I_{i}, \quad i=j=0, \cdots, s, \\
0, \quad j=s-p, \cdots, s-1, i=j+1, \cdots, s, \text { and }(i, j) \neq(s, s-p) .
\end{array}\right.
$$

The matrix $M\left(\varphi_{s-p}\right)$ has the following structure:

$$
M\left(\varphi_{s-p}\right)=\left(\begin{array}{c|c|c|c|c|c|c|c}
1 & 0 & \ldots & 0 & 0 & 0 & 0 & 0 \\
\hline 0 & I_{1} & 0 & 0 & 0 & 0 & 0 & \vdots \\
\hline 0 & 0 & I_{2} & 0 & 0 & 0 & 0 & \vdots \\
\hline \vdots & \vdots & 0 & \ddots & \vdots & \vdots & \vdots & \vdots \\
\hline 0 & B_{p+1,1} & 0 & \ldots & I_{s-p} & 0 & 0 & \vdots \\
\hline 0 & \ldots & B_{p+2,2} & 0 & 0 & I_{s-p+1} & 0 & \vdots \\
\hline 0 & \ldots & \ldots & \ddots & \vdots & 0 & \ddots & 0 \\
\hline 0 & B_{s, 1} & B_{s, 2} & \ldots & B_{s, s-p} & 0 & \ldots & I_{s}
\end{array}\right) .
$$

The entries of $B_{p+1,1}, B_{p+2,2}, \ldots, B_{s, s-p}$ are linear forms in the variables $a_{\underline{i}}^{j}$, with $|\underline{i}|=p+1, j=1, \cdots, n$. We are mainly interested in $B_{s, s-p}$ which is an $\left(\begin{array}{c}n+s-1 \\ s\end{array}\right) \times$ $\left(\begin{array}{c}n+s-p-1 \\ s-p\end{array}\right)$ matrix whose columns correspond to $x^{W}$ with $|W|=s-p$ and the rows correspond to the coefficients of $x^{L}$ with $|L|=s$ in $\varphi\left(x^{W}\right)$. One has

$$
\varphi\left(x^{W}\right)=x^{W}+\sum_{j=1}^{n} w_{j}\left(\sum_{|\underline{i}|=p+1} a_{\underline{i}}^{j} x^{W-\delta_{j}+\underline{i}}\right)+\ldots
$$

where $W=\left(w_{1}, \ldots, w_{n}\right) \in \mathbb{N}^{n}$ with $|W|=s-p$. Here $\delta_{j}$ is the $n$-uple with 0 -entries but 1 in position $j$. We remark that $\left|W-\delta_{j}+\underline{i}\right|=s$. Then the entry of $B_{s, s-p}$ corresponding to the $L$ row, $|L|=s$, and $W$ column, $|W|=s-p$, is

$$
\left(B_{s, s-t}\right)_{L, W}=\sum_{W-\delta_{j}+\underline{i}=L} w_{j} a_{\underline{i}}^{j} .
$$


Let $F, G$ be polynomials of degree $s$ of $P$ and let $\varphi_{s-p}$ be a $K$-algebra isomorphism of type (9) sending $A_{F}$ to $A_{G}$. We denote by $F[j]$ (respectively $G[j]$ ) the homogeneous component of degree $j$ of $F$ (respectively of $G$ ), that is, $F=$ $F[s]+F[s-1]+\ldots(G=G[s]+G[s-1]+\ldots)$.

By (8) we have

$$
[G]_{\Omega^{*}} M\left(\varphi_{s-p}\right)=[F]_{\Omega^{*}}
$$

In particular we deduce

$$
[F[j]]_{\Omega^{*}}= \begin{cases}{[G[s-p]]_{\Omega^{*}}+[G[s]]_{\Omega^{*}} B_{s, s-p},} & j=s-p, \\ {[G[j]]_{\Omega^{*}},} & j=s-p+1, \cdots, s .\end{cases}
$$

We are going to study $[G[s]]_{\Omega^{*}} B_{s, s-p}$. Let $\left[\alpha_{\underline{i}}\right]$ be the vector of the coordinates of $G[s]$ w.r.t. $\Omega^{*}$, i.e.

$$
G[s]=\sum_{|\underline{i}|=s} \alpha_{\underline{i}} \frac{1}{\underline{i} !} y^{\underline{i}} ;
$$

the entries of $[G[s]]_{\Omega^{*}} B_{s, s-p}$ are bi-homogeneous forms in the components of $\left[\alpha_{\underline{i}}\right]$ and $\underline{a}=\left(a_{i}^{1}, \ldots, a_{i}^{n}\right)$ such that $|\underline{i}|=p+1$ of bi-degree $(1,1)$. Hence there exists a matrix $M^{[s-p]}(G[\bar{s}])$ of size $\left(\begin{array}{c}n-1+s-p \\ n-1\end{array}\right) \times n\left(\begin{array}{l}n+p \\ n-1\end{array}\right)$ and entries in the $K\left[\alpha_{\underline{i}}\right]$ such that

$$
{ }^{\tau}\left(\left[\alpha_{\underline{i}}\right] B_{s, s-p}\right)=M^{[s-p]}(G[s]){ }^{\tau} \underline{a}
$$

where ${ }^{\tau} \underline{a}$ denotes the transpose of the row-vector $\underline{a}$. We are going to describe the entries of $M^{[s-p]}(G[s])$. We label the columns of $M^{[s-p]}(G[s])$ with the set of indexes $(j, \underline{i}), j=1, \cdots, n,|\underline{i}|=p+1$, corresponding to the entries of $\underline{a}=\left(a_{\underline{i}}^{1},|\underline{i}|=\right.$ $\left.p+1 ; \cdots ; a_{\underline{i}}^{n},|\underline{i}|=p+1\right) \in K^{n\left(\begin{array}{c}n+p \\ n-1\end{array}\right)}$.

Lemma 2.1. The entry of $M^{[s-p]}(G[s])$ corresponding to the $W$ row, $|W|=s-p$, and column $(j, \underline{i}) \in\{1, \cdots, n\} \times\{\underline{i} ;|\underline{i}|=p+1\}$ is

$$
M^{[s-p]}(G[s])_{W,(j, \underline{i})}=w_{j} \alpha_{W-\delta_{j}+\underline{i}} .
$$

Proof. Given $W,|W|=s-p$, the coordinate of $[G[s]]_{\Omega^{*}} B_{s, s-p}$ with respect to $\left(x^{W}\right)^{*}$ is, by (10),

$$
\begin{aligned}
\left([G[s]]_{\Omega^{*}} B_{s, s-p}\right)_{\left(x^{W}\right)^{*}} & =\sum_{|L|=s}\left(B_{s, s-p}\right)_{W, L} \alpha_{L} \\
& =\sum_{|L|=s}\left(\sum_{W-\delta_{j}+\underline{i}=L} w_{j} a_{\underline{i}}^{j}\right) \alpha_{L}=\sum_{j,|\underline{i}|=p+1} w_{j} \alpha_{W-\delta_{j}+\underline{i}} a_{\underline{i}}^{j}
\end{aligned}
$$

so the entry of $M^{[s-p]}(G[s])$ corresponding to the $W$ row, $|W|=s-p$, and column $(j, \underline{i}) \in\{1, \cdots, n\} \times\{\underline{i} ;|\underline{i}|=p+1\}$ is $w_{j} \alpha_{W-\delta_{j}+\underline{i}}$.

The goal is now to present a structure of $M^{[s-p]}(G[s])$ in terms of $G[s]$. In particular we will prove that the rank of $M^{[s-p]}(G[s])$ can be expressed in terms of the Hilbert function of $A_{G[s]}$. We need further notation.

For every $i=1, \cdots, n$, we denote $S_{p}^{i}$ the set of monomials $x^{\alpha}$ of degree $p$ such that $x^{\alpha} \in x_{i}\left(x_{i}, \cdots, x_{n}\right)^{p-1}$, hence $\#\left(S_{p}^{i}\right)=\left(\begin{array}{c}p-1+n-i \\ p-1\end{array}\right)$. By definition $S_{p}^{1} \cup \cdots \cup S_{p}^{n}$ 
is the set of monomials of degree $p$ and

$$
\text { the last }\left(\begin{array}{c}
p-2+n-i \\
p-1
\end{array}\right) \text { elements of } S_{p}^{i} \text { correspond to } \frac{x_{i}}{x_{i+1}} S_{p}^{i+1},
$$

For instance $S_{3}^{1}=\left\{x_{1}^{3}, x_{1}^{2} x_{2}, x_{1}^{2} x_{3}, x_{1} x_{2}^{2}, x_{1} x_{2} x_{3}, x_{1} x_{3}^{2}\right\}, S_{3}^{2}=\left\{x_{2}^{3}, x_{2}^{2} x_{3}, x_{2} x_{3}^{2}\right\}$, $S_{3}^{3}=\left\{x_{3}^{3}\right\}$. We write $\log \left(x^{\alpha}\right)=\alpha$ for all $\alpha \in \mathbb{N}^{n}$.

Lemma 2.2. The matrix $M^{[s-p]}(G[s])$ has the following upper-diagonal structure:

$$
M^{[s-p]}(G[s])=\left(\begin{array}{l|l|l|l|l}
M_{1} & * & \cdots & * & * \\
\hline 0 & M_{2} & \cdots & * & * \\
\hline \vdots & \vdots & \vdots & \vdots & \vdots \\
\hline 0 & 0 & 0 & M_{n-1} & * \\
\hline 0 & 0 & 0 & 0 & M_{n}
\end{array}\right)
$$

where $M_{j}$ is a matrix of size $\left(\begin{array}{c}s-p-1+n-j \\ s-p-1\end{array}\right) \times\left(\begin{array}{l}n+p \\ n-1\end{array}\right), j=1, \cdots, n$, defined as follows: the entries of $M_{j}$ are the entries of $M^{[s-p]}(G[s])$ corresponding to the rows $W \in$ $\log \left(S_{s-p}^{j}\right)$ and columns $(j, \underline{i}),|\underline{i}|=p+1$. We label the entries of $M_{j}$ with respect to these multi-indices. Then it holds:

(i) for all $W=\left(w_{1}, \cdots, w_{n}\right) \in \log \left(S_{s-p}^{1}\right)$ and $\underline{i},|\underline{i}|=p+1$,

$$
w_{1} \Delta^{p+1}(G[s])_{\left(W-\delta_{1}, \underline{i}\right)}=M_{1(W,(1, \underline{i}))},
$$

(ii) for all $j=1, \cdots, n-1, W \in \log \left(S_{s-p}^{j+1}\right)$,

$$
M_{j+1,(W,(j+1, *))}=w_{j+1} M_{j,(L,(j, *))}
$$

with $L=\delta_{j}+W-\delta_{j+1}$,

Proof. First we prove that $M^{[s-p]}(G[s]$ has the upper-diagonal structure as in the claim. Since the entry of $M^{[s-p]}(G[s])$ corresponding to the $W$ row, $|W|=s-p$, and column $(j, \underline{i}) \in\{1, \cdots, n\} \times\{\underline{i} ;|\underline{i}|=p+1\}$ is $w_{j} \alpha_{W-\delta_{j}+\underline{i}}$, this entry is zero if $W \in \log \left(S_{s-p}^{t}\right)$ and $j<t \leq n$.

(i) Notice that the set of multi-indices $L=W-\delta_{1},|W|=s-p$, agrees with the set of multi-indices of degree $s-p-1$; see (14). Hence by Proposition 1.6 and Lemma 2.1 we have

$$
w_{1} \Delta^{p+1}(G[s])_{\left(W-\delta_{1}, \underline{i}\right)}=w_{1} \alpha_{W-\delta_{1}+\underline{i}}=M_{1(W,(1, \underline{i}))} .
$$

(ii) If $|\underline{i}|=p+1$ and $L=\delta_{j}+W-\delta_{j+1}$, then, by Lemma 2.1.

$$
M_{j+1,(W,(j+1, *))}=w_{j+1} \alpha_{W-\delta_{j+1}+\underline{i}}=w_{j+1} \alpha_{L-\delta_{j}+\underline{i}}=w_{j+1} M_{j,(L,(j, *))} .
$$

As a consequence of $(i)$ and $(i i)$, the matrix $\Delta^{p+1}(G[s])$ is strongly involved in the computation of the rank of $M^{[s-p]}(G[s])$.

Corollary 2.3. If $s \leq 4$ then $\operatorname{rank}\left(M^{[s-p]}(G[s])\right)$ is maximal if and only if $\operatorname{rank}\left(\Delta^{p+1}(G[s])\right)$ is maximal.

Proof. Notice that $M^{[s-p]}(G[s])$ has an upper-diagonal structure where the rows of the diagonal blocks $M_{j}$ are a subset of the rows of the first block matrix $M_{1}$. Let us assume that the number of rows of $M_{1}$ is not larger than the number of columns of $M_{1}$. As a consequence the same holds for $M_{j}$ with $j>1$. Then we can compute the rank of $M^{[s-p]}(G[s])$ by rows, so $\operatorname{rank}\left(M^{[s-p]}(G[s])\right)$ is maximal if and only if $\operatorname{rank}\left(\Delta^{p+1}(G[s])\right)$ is maximal. Since $M_{1}$ is an $\left(\begin{array}{c}s-p-2+n \\ s-p-1\end{array}\right) \times\left(\begin{array}{l}n+p \\ n-1\end{array}\right)$ matrix, if 
$\left(\begin{array}{c}n+s-p-2 \\ s-p-1\end{array}\right)=\left(\begin{array}{c}n+s-p-2 \\ n-1\end{array}\right) \leq\left(\begin{array}{c}n+p \\ n-1\end{array}\right)$, we get the result. This inequality is equivalent to $n+s-p-2 \leq n+p$, i.e. $s \leq 2 p+2$, since $p \geq 1$ we get that $s \leq 4$.

Example 3.4 shows that Corollary 2.3 fails for $s=5$.

We may generalize the previous facts to a sequence $\underline{G}=G_{1}, \ldots, G_{t}$ of polynomials of degree $s$ of $P$. Let $\varphi_{s-p}$ be a $K$-algebra isomorphism of type (9) sending $A_{\underline{F}}$ to $A_{\underline{G}}$ where $\underline{F}=F_{1}, \ldots, F_{t}$. In particular we assume that, as in (11),

$$
\left[G_{r}\right]_{\Omega^{*}} M\left(\varphi_{s-p}\right)=\left[F_{r}\right]_{\Omega^{*}},
$$

for every $r=1, \ldots, t$. We deduce the analogous of (12) and we restrict our interest to

where

$$
[\underline{G}[s]]_{\Omega^{*}} B_{s, s-p}^{\oplus t}
$$

$$
B_{s, s-p}^{\oplus t}:=\left(\begin{array}{c}
\frac{B_{s, s-p}}{\vdots} \\
\frac{B_{s, s-p}}{a_{s-s}}
\end{array}\right)
$$

obtained by gluing $t$ times the matrix $B_{s, s-p}$ and where $[\underline{G}[s]]_{\Omega^{*}}$ is the row $\left(\left[G_{r}[s]\right]_{\Omega^{*}}\right.$ $: r=1, \ldots, t)$. Accordingly with (13), we define the matrix $M^{[s-p]}\left(G_{r}[s]\right)$ of size $\left(\begin{array}{c}n-1+s-p \\ n-1\end{array}\right) \times n\left(\begin{array}{l}n+p \\ n-1\end{array}\right)$ and entries depending on $[\underline{G}[s]]_{\Omega^{*}}$ such that

If we define

$$
{ }^{\tau}\left(\left[G_{r}[s]\right]_{\Omega^{*}} B_{s, s-p}\right)=M^{[s-p]}\left(G_{r}[s]\right)^{\tau} \underline{a} .
$$

$$
M^{[s-p]}(\underline{G}[s]):=\left(\begin{array}{c}
\frac{M^{[s-p]}\left(G_{1}[s]\right)}{\vdots} \\
\left.\frac{M^{[s-p]}\left(G_{t}[s]\right)}{s}\right)
\end{array}\right.
$$

which is a $t\left(\begin{array}{c}n-1+s-p \\ n-1\end{array}\right) \times n\left(\begin{array}{l}n+p \\ n-1\end{array}\right)$ matrix, we get

$$
\tau\left([\underline{G}[s]]_{\Omega^{*}} B_{s, s-p}^{\oplus t}\right)=M^{[s-p]}(\underline{G}[s])^{\tau} \underline{a} .
$$

The matrix $M^{[s-p]}(\underline{G}[s])$ has the same shape of $M^{[s-p]}(G[s])$, already described in Lemma 2.2 and its blocks correspond to suitable submatrices of $\left(\Delta^{p+1}(\underline{G}[s])\right)$ (see (6) ). Hence we have an analogue to (12) for the level case

$$
\left[F_{r}[j]\right]_{\Omega^{*}}= \begin{cases}{\left[G_{r}[s-p]\right]_{\Omega^{*}}+\underline{a}^{\tau}\left(M^{[s-p]}(\underline{G}[s])\right),} & j=s-p, \\ {\left[G_{r}[j]\right]_{\Omega^{*}},} & j=s-p+1, \cdots, s\end{cases}
$$

for all $r=1, \ldots, t$.

\section{Compressed Gorenstein Algebras}

Let $A=A_{G}$ be a compressed Gorenstein local $K$-algebra of socle degree $s$. We recall that, by Proposition 1.8 if $G=G[s]+G[s-1]+\ldots$, then

$$
h_{i}(A)=\operatorname{rank}\left(\Delta^{i}(G[s])=\min \left\{\left(\begin{array}{c}
n-1+i \\
n-1
\end{array}\right),\left(\begin{array}{c}
n-1+s-i \\
n-1
\end{array}\right)\right\}\right.
$$

for every $i=1, \ldots, s$.

Theorem 3.1. Let $A$ be an Artin compressed Gorenstein local $K$-algebra. If $s \leq 4$, then $A$ is canonically graded. 
Proof. Let $A$ be an Artin compressed Gorenstein local $K$-algebra of socle degree $s \geq 2$ and embedding dimension $n$. Then $A=A_{G}$ with $G \in P=K\left[y_{1}, \ldots, y_{n}\right]$ a polynomial of degree $s$ and $g r_{\mathfrak{m}}(A)=P / A n n(G[s])$ is a compressed Gorenstein graded algebra of socle degree $s \geq 2$ and embedding dimension $n$ (see Proposition 1.4).

The main result of $[6$ shows that if $s \leq 3$, then $A$ is canonically graded. Let us assume $s=4$. Then the Hilbert function is $\left\{1, n,\left(\begin{array}{c}n+1 \\ 2\end{array}\right), n, 1\right\}$. Because $A_{G[4]}$ is a compressed Gorenstein algebra with the same Hilbert function of $A$, we may assume $G=G[4]+G[3]$. In fact $P_{1}, P_{2} \subseteq\langle G[4]\rangle_{R}$ because of (3) and, as a consequence, it is easy to see that $\langle G[4]+G[3]\rangle_{R}=\langle G[4]+G[3]+G[2]+\ldots\rangle_{R}$.

So we have to prove that, however we fix $G[3]$, there exists an automorphism $\varphi \in \operatorname{Aut}\left(R / \mathcal{M}^{5}\right)$ such that

$$
A_{G} \simeq A_{G[4]} .
$$

We consider for every $j=1, \ldots, n$

$$
\varphi_{3}\left(x_{j}\right)=x_{j}+\sum_{|\underline{i}|=2} a_{\underline{i}}^{j} x^{\underline{i}}+\text { higher terms. }
$$

If $A_{F}=\varphi_{3}^{-1}\left(A_{G}\right)$, then from (12) and (13) we get

$$
\begin{aligned}
& {[F[3]]_{\Omega^{*}}=[G[3]]_{\Omega^{*}}+\underline{a}^{\tau}\left(M^{[3]}(G[4])\right)} \\
& {[F[4]]_{\Omega^{*}}=[G[4]]_{\Omega^{*}}}
\end{aligned}
$$

where $\underline{a}=\left(a_{\underline{i}}^{1}, \ldots, a_{\underline{i}}^{n}\right)$. By Proposition 1.8 and Corollary 2.3, we know that the matrix $M^{[3]}(\bar{G}[4])$ has maximal rank and it coincides with the number of rows, so there exists a solution $\underline{a} \in K^{n}$ of (18) such that $F[3]=0$ and $F[4]=G[4]$.

Let $A$ be a local $K$-algebra of embedding dimension $n$ and socle degree $s$. By looking at the dual module, it is clear that if $s \leq 2$, then $A$ is graded because the dual module can be generated by homogeneous polynomials of degree at least 2. The aim is now to list large classes of local compressed algebras of embedding dimension $n$, socle degree $s$ and socle type $E=\left(0, \ldots, e_{v-1}, e_{v}, \ldots, e_{s}, 0,0, \ldots\right)$ which are canonically graded. Examples will prove that the following result cannot be extended to higher socle degrees.

Theorem 3.2. Let $A$ be an Artin compressed $K$-algebra of embedding dimension $n$, socle degree $s$ and socle type $E$. Then $A$ is canonically graded in the following cases:

(1) $s \leq 3$,

(2) $s=4$ and $e_{4}=1$,

(3) $s=4$ and $n=2$.

Proof. Since a local ring with Hilbert function $\{1, n, t\}$ is always graded (the dual module can be generated by quadratic forms), we may assume $s \geq 3$.

If $s=3$ and $A$ is a compressed level algebra, then $A$ is canonically graded by [4]. If $A$ is not necessarily level, but compressed, then by (11) the socle type is $\left\{0,0, e_{2}, e_{3}\right\}$ and the Hilbert function is $\left\{1, n, h_{2}, e_{3}\right\}$ where

$$
h_{2}=\min \left\{\operatorname{dim}_{K} R_{2}, e_{2}+e_{3} n\right\} .
$$


Because $g r_{\mathfrak{m}}(A)=P / I^{*}$ has embedding dimension $n, P_{\leq 1} \subseteq\left(I^{*}\right)^{\perp}$. Then we may assume that in any system of coordinates $I^{\perp}$ is generated by $e_{2}$ quadratic forms and $e_{3}$ polynomials $G_{1}, \ldots, G_{e_{3}}$ of degree 3 . Then the result follows because $R / A n n_{R}\left(G_{1}, \ldots, G_{e_{3}}\right)$ is a 3-level compressed algebra of type $e_{3}$ and hence canonically graded.

Let us assume $s=4$ and $e_{4}=1$. We recall that if $A$ is Gorenstein, then the result follows by Theorem 3.1. Since $A$ is compressed, by (11) the socle type is $\left(0,0,0, e_{3}, 1\right)$. This means that $I^{\perp}$ is generated by $e_{3}$ polynomial of degree 3 and one polynomial of degree 4 . Similarly to the above part, because $P_{\leq 2} \subseteq\left(I^{*}\right)^{\perp}, I^{\perp}$ can be generated by $e_{3}$ forms of degree 3 and one polynomial of degree 4 . As before the problem is reduced to the Gorenstein case with $s=4$ and the result follows.

Assume $s=4$ and $n=2$. If $e_{4}=1$, then we are in case (2). If $e_{4}>1$, because $A$ is compressed, the possible socle types are: $E_{i}=(0,0,0,0, i)$ with $i=2, \cdots, 5$ and since $A$ is compressed, the corresponding Hilbert function is $\{1,2,3,4, i\}$. In each case $A$ is graded because the Hilbert function forces the dual module to be generated by forms of degree 4 .

The following example shows that Theorem 3.1 fails if $A$ is Gorenstein of socle degree $s=4$, but not compressed, i.e. the Hilbert function is not maximal.

Example 3.3. Let $A$ be an Artin Gorenstein local $K$-algebra with Hilbert function $H F_{A}=\{1,2,2,2,1\}$. The local ring is called almost stretched and a classification can be found in [7]. In this case $A$ is isomorphic to one and only one of the following rings:

(a) $A=R / I$ with $I=\left(x_{1}^{4}, x_{2}^{2}\right) \subseteq R=K\left[\left[x_{1}, x_{2}\right]\right]$, and $I^{\perp}=\left\langle y_{1}^{3} y_{2}\right\rangle$. In this case $A$ is canonically graded.

(b) $A=R / I$ with $I=\left(x_{1}^{4},-x_{1}^{3}+x_{2}^{2}\right) \subseteq R=K\left[\left[x_{1}, x_{2}\right]\right]$, and $I^{\perp}=\left\langle y_{1}^{3} y_{2}+y_{2}^{3}\right\rangle$. The associated graded ring is of type $(a)$ and it is not isomorphic to $R / I$. Hence $A$ is not canonically graded.

(c) $A=R / I$ with $I=\left(x_{1}^{2}+x_{2}^{2}, x_{2}^{4}\right) \subseteq R=K\left[\left[x_{1}, x_{2}\right]\right]$, and $I^{\perp}=\left\langle y_{1} y_{2}\left(y_{1}^{2}-y_{2}^{2}\right)\right\rangle$. In this case $A$ is graded.

The following example shows that Theorem 3.1 cannot be extended to compressed Gorenstein algebras of socle degree $s=5$.

Example 3.4. Let us consider the ideal

$$
I=\left(x_{1}^{4}, x_{2}^{3}-2 x_{1}^{3} x_{2}\right) \subset R=K\left[\left[x_{1}, x_{2}\right]\right] .
$$

The quotient $A=R / I$ is a compressed Gorenstein algebra with $H F_{A}=\{1,2,3,3$, $2,1\}, I^{*}=\left(x_{1}^{4}, x_{2}^{3}\right)$ and $I^{\perp}=\left\langle y_{1}^{3} y_{2}^{2}+y_{2}^{4}\right\rangle$. We will prove that $I$ is not isomorphic to $I^{*}$. Assume that there exists an analytic isomorphism $\varphi$ of $R$ mapping $I$ into $I^{*}$. It is easy to see that Jacobian matrix of $\varphi$ is diagonal because $\left(I^{*}\right)^{\perp}=\left\langle y_{1}^{3} y_{2}^{2}\right\rangle$. We perform the computations modulo $\left(x_{1}, x_{2}\right)^{5}$, so we only have to consider the following coefficients of $\varphi$ :

$$
\left\{\begin{array}{l}
\varphi\left(x_{1}\right)=a x_{1}+\ldots \\
\varphi\left(x_{2}\right)=b x_{2}+i x_{1}^{2}+j x_{1} x_{2}+k x_{2}^{2}+\ldots
\end{array}\right.
$$

where $a, b$ are units, $i, j, k \in K$. After the isomorphism $x_{1} \rightarrow 1 / a x_{1}, x_{2} \rightarrow 1 / b x_{2}$, we may assume $a=b=1$. Then we have

$$
I^{*}=\varphi(I)=\left(x_{1}^{4}, x_{2}^{3}-2 x_{1}^{3} x_{2}+3 i x_{1}^{2} x_{2}^{2}+3 j x_{1} x_{2}^{3}+3 k x_{2}^{4}\right) \quad \text { modulo }\left(x_{1}, x_{2}\right)^{5} .
$$


Hence there exist $\alpha \in K, \beta \in R$ such that

$$
x_{2}^{3}-2 x_{1}^{3} x_{2}+3 i x_{1}^{2} x_{2}^{2}+3 j x_{1} x_{2}^{3}+3 k x_{2}^{4}=\alpha x_{1}^{4}+\beta x_{2}^{3} \quad \text { modulo }\left(x_{1}, x_{2}\right)^{5} .
$$

From this equality we deduce $\alpha=0$ and

$$
2 x_{1}^{3} x_{2}=x_{2}^{2}\left(x_{2}+3 i x_{1}^{2}+3 j x_{1} x_{2}+3 k x_{2}^{2}-\beta x_{2}\right) \quad \text { modulo }\left(x_{1}, x_{2}\right)^{5},
$$

a contradiction, so $I$ is not isomorphic to $I^{*}$.

It is interesting to notice that we can get the same conclusion by following the line of the proof of Theorem 3.1 Let $\varphi$ as above sending $I$ into $I^{*}$. If we denote by $\left(z_{i}\right)_{i=1, \ldots, 6}$ the coordinates of a homogeneous form $G[5]$ of degree 5 in $y_{1}, y_{2}$ with respect $\Omega^{*}$, then by Lemma 2.1. the matrix $M^{[4]}(G[5])(s=5, p=1)$ has the following shape:

$$
\left(\begin{array}{cccccc}
4 z_{1} & 4 z_{2} & 4 z_{3} & 0 & 0 & 0 \\
3 z_{2} & 3 z_{3} & 3 z_{4} & z_{1} & z_{2} & z_{3} \\
2 z_{3} & 2 z_{4} & 2 z_{5} & 2 z_{2} & 2 z_{3} & 2 z_{4} \\
z_{4} & z_{5} & z_{6} & 3 z_{3} & 3 z_{4} & 3 z_{5} \\
0 & 0 & 0 & 4 z_{4} & 4 z_{5} & 4 z_{6}
\end{array}\right)
$$

In our case $G[5]=y_{1}^{3} y_{2}^{2}$, so all $z_{i}$ are zero but $z_{3}=12$, hence the above matrix has rank 4 and it has not maximal rank accordingly with Corollary 2.3 . Since all the rows are not zero except the last one, it is easy to see that $F[4]=y_{2}^{4}$ is not in the image of $M^{[4]}(G[5])$, as (12) requires.

The following example shows that Theorem 3.2 cannot be extended to compressed type 2 level algebras of socle degree $s=4$.

Example 3.5. Let us consider the forms $G_{1}[4]=y_{1}^{2} y_{2} y_{3}, G_{2}[4]=y_{1} y_{2}^{2} y_{3}+y_{2} y_{3}^{3}$ in $P=K\left[y_{1}, y_{2}, y_{3}\right]$ of degree 4 and define in $R=K\left[\left[x_{1}, x_{2}, x_{3}\right]\right]$ the ideal

$$
I=\operatorname{Ann}\left(G_{1}[4]+y_{3}^{3}, G_{2}[4]\right) .
$$

Then $A=R / I$ is a compressed level algebra with socle degree 4, type 2 and Hilbert function $H F_{A}=\{1,3,6,6,2\}$. We prove that $A$ is not canonically graded.

We know that $I^{*}=\operatorname{Ann}\left(G_{1}[4], G_{2}[4]\right)$ and we prove that $A$ and $g r_{\mathfrak{m}}(A)$ are not isomorphic as $K$-algebras. Let $\varphi$ be an analytic isomorphism sending $I$ to $I^{*}$. Then it is easy to see that $\varphi=I_{3}$ modulo $\left(x_{1}, x_{2}, x_{3}\right)^{2}$. Following the approach of this paper, we compute the matrix $M^{[3]}\left(G_{1}[4], G_{2}[4]\right)$ of size $20 \times 18$ and, accordingly with (12), we show that $y_{3}^{3}$ is not in the image of $M^{[3]}\left(G_{1}[4], G_{2}[4]\right)$.

Let $F_{1}[4], F_{2}[4]$ be two homogeneous forms of degree 4 of $R=K\left[y_{1}, y_{2}, y_{3}\right]$. We denote by $\left(z_{i}^{j}\right)_{i=1, \ldots, 15}$ the coordinates of $F_{j}[4]$ with respect to the basis $\Omega^{*}, j=1,2$. Then the $20 \times 18$ matrix $M^{[3]}\left(F_{1}[4], F_{2}[4]\right)$ has the following shape (see (15)). 


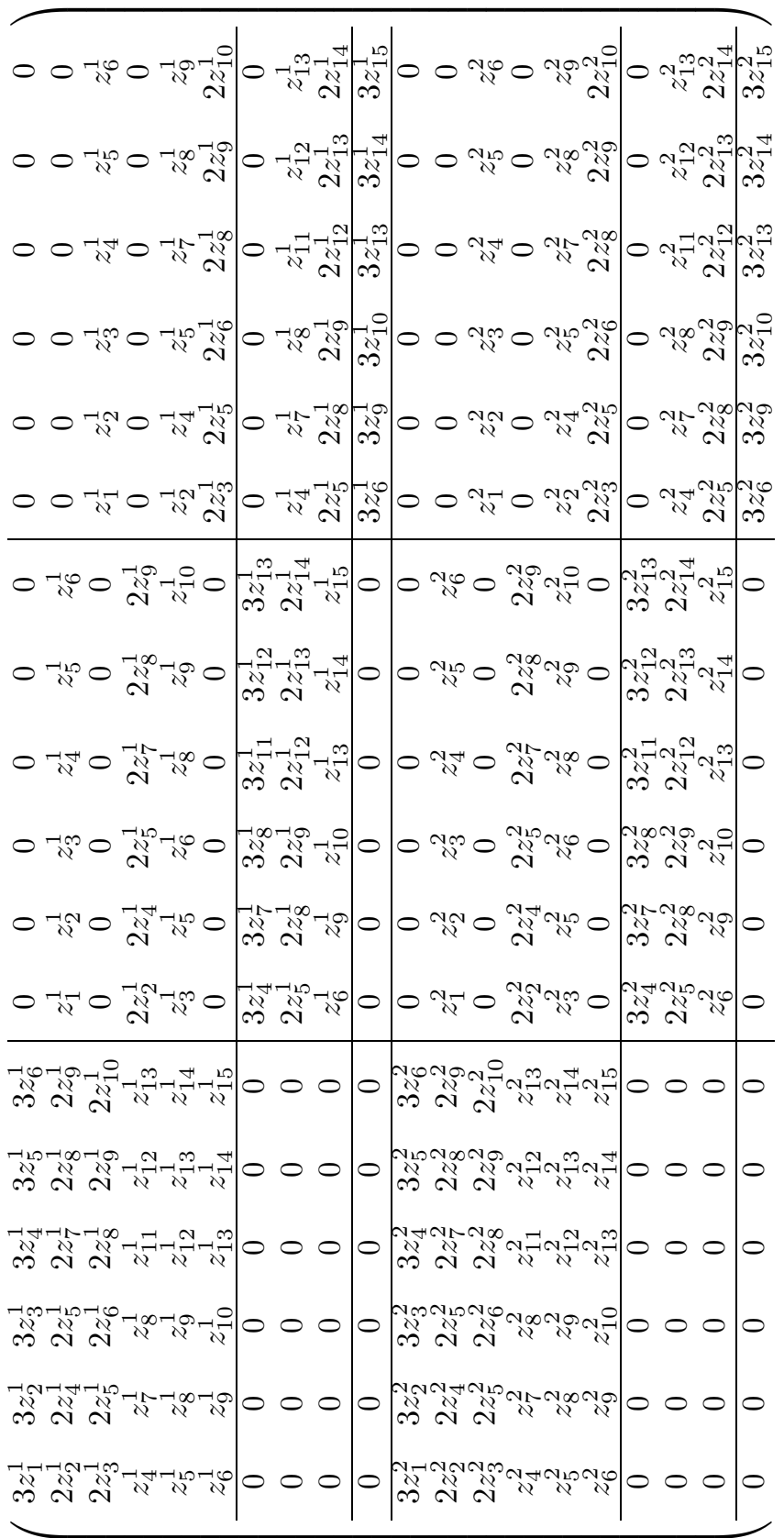


It is enough to specialize the matrix to our case for proving that $y_{3}^{3}$ is not in the image of $M^{[3]}\left(G_{1}[4], G_{2}[4]\right)$.

Remark 3.6. Let $\mathcal{C}_{s, t}$ be the family of level Artin algebras of socle degree $s$ and type $t$. This family can be parameterized by a non-empty open Zariski subset $\mathcal{I}_{s, t}$ of the affine space of $t$-uples of degree $s$ polynomials of $P$. The previous examples suggest that, by dimensional reasons, if $s, t$ are not in the hypothesis of Theorem 3.2 , then a generic element $F \in \mathcal{I}_{s, t}$ defines a non-canonically graded compressed level algebra $R / A n n_{R}(\langle F\rangle)$ of socle degree $s$ and type $t$.

\section{REFERENCES}

[1] Mats Boij, Betti numbers of compressed level algebras, J. Pure Appl. Algebra 134 (1999), no. 2, 111-131, DOI 10.1016/S0022-4049(97)90163-8. MR.1663785 (99m:13023)

[2] Gianfranco Casnati, Juan Elias, Roberto Notari, and Maria Evelina Rossi, Poincaré series and deformations of Gorenstein local algebras, Comm. Algebra 41 (2013), no. 3, 1049-1059, DOI 10.1080/00927872.2011.636643. MR3037178

[3] Young Hyun Cho and Anthony Iarrobino, Hilbert functions and level algebras, J. Algebra 241 (2001), no. 2, 745-758, DOI 10.1006/jabr.2001.8787. MR.1843323(2002f:13032)

[4] A. De Stefani, Short Artinian level local k-algebras, Comm. Algebra 42 (2014), no. 2, 729-754.

[5] J. Elias and A. Iarrobino, The Hilbert function of a Cohen-Macaulay local algebra: extremal Gorenstein algebras, J. Algebra 110 (1987), no. 2, 344-356, DOI 10.1016/00218693(87)90050-0. MR910388 (88i:13029)

[6] J. Elias and M. E. Rossi, Isomorphism classes of short Gorenstein local rings via Macaulay's inverse system, Trans. Amer. Math. Soc. 364 (2012), no. 9, 4589-4604, DOI 10.1090/S00029947-2012-05430-4. MR2922602

[7] J. Elias and G. Valla, Isomorphism classes of certain Artinian Gorenstein Algebras, Algebra and Representation Theory 14 (2011), 429-448.

[8] Jacques Emsalem, Géométrie des points épais (French, with English summary), Bull. Soc. Math. France 106 (1978), no. 4, 399-416. MR518046 (80j:14008)

[9] R. Fröberg and D. Laksov, Compressed algebras, Complete intersections (Acireale, 1983), Lecture Notes in Math., vol. 1092, Springer, Berlin, 1984, pp. 121-151, DOI 10.1007/BFb0099360. MR775880 (86f:13012)

[10] Anthony Iarrobino, Compressed algebras: Artin algebras having given socle degrees and maximal length, Trans. Amer. Math. Soc. 285 (1984), no. 1, 337-378, DOI 10.2307/1999485. MR748843 (85j:13030)

[11] Anthony A. Iarrobino, Associated graded algebra of a Gorenstein Artin algebra, Mem. Amer. Math. Soc. 107 (1994), no. 514, viii+115, DOI 10.1090/memo/0514. MR1184062 (94f:13009)

[12] F. S. Macaulay, The algebraic theory of modular systems, Cambridge Mathematical Library, Cambridge University Press, Cambridge, 1994. Revised reprint of the 1916 original; With an introduction by Paul Roberts. MR,1281612 (95i:13001)

Departament D’Àlgebra i Geometria, Facultat de Matemàtiques, Universitat de Barcelona, Gran Via 585, 08007 Barcelona, Spain

E-mail address: elias@ub.edu

Dipartimento di Matematica, Università di Genova, Via Dodecaneso 35, 16146 Genova, ITALY

E-mail address: rossim@dima.unige.it 